\title{
BMJ Open Switching antipsychotics to support the physical health of people with severe mental illness: a qualitative study of healthcare professionals' perspectives
}

\author{
Annabel Nash, ${ }^{1,2}$ Tom Kingstone (D) , , 3 Saeed Farooq, ${ }^{1,3}$ Jessica Tunmore, ${ }^{1,3}$ \\ Carolyn A Chew-Graham ${ }^{1,3}$
}

To cite: Nash A, Kingstone T, Farooq S, et al. Switching antipsychotics to support the physical health of people with severe mental illness: a qualitative study of healthcare professionals' perspectives. BMJ Open 2021;11:e042497. doi:10.1136/ bmjopen-2020-042497

- Prepublication history and supplemental material for this paper is available online. To view these files, please visit the journal online (http://dx.doi. org/10.1136/bmjopen-2020042497).

Received 06 July 2020 Revised 22 January 2021 Accepted 08 February 2021

Check for updates

(c) Author(s) (or their employer(s)) 2021. Re-use permitted under CC BY-NC. No commercial re-use. See rights and permissions. Published by BMJ.

${ }^{1}$ Research and Innovation Department, Midlands Partnership NHS Foundation Trust, Stafford, UK ${ }^{2}$ School of Life Sciences and Education, Staffordshire University, Stoke-on-Trent, UK

${ }^{3}$ School of Medicine, Keele University, Keele, UK

Correspondence to Dr Tom Kingstone;

t.kingstone@keele.ac.uk

\section{ABSTRACT}

Objectives The side effects of antipsychotic medications (APs) can increase the risk of developing physical health conditions. Some APs pose a higher risk than others. Evidence suggests switching to lower risk APs can support physical health outcomes. We sought to explore the views of healthcare professionals about switching antipsychotics to support physical health in people with severe mental illness (SMI).

Design A qualitative study with semi-structured interviews conducted with general practitioners (GPs), psychiatrists and mental health nurses. The main focus was to explore participants' views on the physical health of people with SMI, the impact of APs and decision-making about switching medication to support physical health. Data were analysed thematically using principles of constant comparison.

Settings Participants recruited through primary care and one mental health trust in the West Midlands.

Participants Interviews were conducted with $9 \mathrm{GPs}, 10$ psychiatrists and 4 mental health nurses.

Results Awareness and knowledge of AP side-effects and risk profiles varied considerably between primary and secondary care clinicians. GPs reported limited awareness, while psychiatrists and nurses demonstrated a comprehensive understanding of AP risk profiles and side-effects. Mental health and control of symptoms were prioritised. Switching to promote physical health was considered as a reactive intervention, once side-effects or complications developed. There were a range of views over where responsibility lay for monitoring physical health and consideration of switching. Collaboration between primary and secondary care was identified as a way to address this.

Conclusions This study presents multidisciplinary perspectives on awareness, decision making, perceived responsibility and barriers to switching APs to support physical health. Collaborative approaches that involve liaison between primary and secondary care, but tailored to the individual patient, may support switching, and present an opportunity to intervene to address the physical health inequalities experienced by individuals with SMI.
Strengths and limitations of this study

- This study addresses a gap in the literature by using qualitative methods to provide an in-depth exploration of healthcare professionals' (HCPS) views and experiences of switching.

- This study explores the perspectives of HCPs from three disciplines (general practice, nursing and psychiatry) to provide a holistic account of perspectives on switching.

- Patients and carers contributed to the study design and interpretation of findings.

- Only 4 mental health nurses from the 53 invited to participate were interviewed which meant data saturation was not achieved for this group. The transferability of the findings to other settings may be restricted due to sampling psychiatrists and mental health nurses from one mental health trust; a more diverse sample, from a range of trusts, might have uncovered different perspectives.

\section{INTRODUCTION}

The life expectancy for people with severe mental illness (SMI) is reduced on average by 15-20 years compared with the general population. ${ }^{1}$ This mortality gap is widening ${ }^{2}$ and continues to be driven by high suicide rates and comorbid physical health problems such as cardiovascular disease (CVD) and diabetes. Individuals with SMI are twice as likely to develop type 2 diabetes compared with the general population. ${ }^{3}$ Poor socioeconomic conditions and lifestyle choices contribute to the increased risk of CVD and diabetes. ${ }^{4-6}$ The National Health Service (NHS) Five Year Forward View stresses the importance of meeting the physical health needs of people with SMI to reduce mortality rates. ${ }^{7}$ To achieve this, we must understand the modifiable factors contributing to this inequality.

Lifestyle factors including poor diet, physical inactivity, smoking and substance misuse contribute to increased cardiometabolic risk. 
However, trials of lifestyle interventions including individuals with SMI prescribed antipsychotic medications (APs) have been largely ineffective, both in terms of improving physical health outcomes and cost-effectiveness. ${ }^{8} 9$ APs can cause increased appetite, weight gain and insulin resistance, all of which increase the risk of metabolic syndrome and contribute to increased morbidity and mortality. Evidence suggests a holistic approach to intervention which addresses the multiple contextual factors impacting upon the physical health of individuals with SMI is required. ${ }^{10}$

Meta-analyses have shown small differences in effectiveness in managing symptoms of psychosis between APs, but significant differences in metabolic side-effects. ${ }^{11-13}$ Considering some of the most commonly prescribed second-generation APs, ${ }^{14}$ clozapine and olanzapine have the highest propensity to cause weight gain and metabolic abnormalities, quetiapine, followed by risperidone have a moderate metabolic risk. In contrast, aripiprazole and lurasidone have the smallest impact on metabolic profiles. ${ }^{11-13}$ A systematic review reported that switching from olanzapine to aripiprazole was associated with weight loss and improved glucose and lipid profiles; no significant differences in mental state were reported for those who switched, suggesting an overall benefit of switching. However, methodological limitations were noted in the studies reviewed. ${ }^{15}$ Guidelines recommend considering a switch of AP as a treatment option to improve physical health. ${ }^{10}$ However, this rarely happens in clinical practice.

The National Institute for Health and Care Excellence recommends that APs for people with SMI should be initiated in specialist care. ${ }^{16}$ Primary care is responsible for conducting screening and physical health interventions for people with SMI on general practitioner (GP) registers. ${ }^{17}$ Over half of people with SMI on a GP list are looked after solely by the practice, without any specialist care input.

Switching of APs could feasibly take place in primary or specialist care. No research has previously explored whether and/or how switching APs to improve physical health in people with SMI is considered or carried out in clinical practice. Our qualitative study aimed to address this gap by exploring healthcare professionals' (HCPs) views on the barriers and facilitators to switching APs.

\section{METHODS}

\section{Design and setting}

A qualitative study in which semi-structured interviews were conducted with HCPs (GPs, psychiatrists and mental health nurses) to explore their views on switching APs to support physical health outcomes for people with SMI. This method supported an in-depth exploration of experiences in this underexplored area. ${ }^{18}$ The study was conducted in primary and secondary care in the West Midlands and North West of England, in urban, semi-rural and rural areas. The 'Consolidated criteria for Reporting

\section{Box 1 Topic guide summary}

Experiences of prescribing antipsychotics

- Prescribing experience.

- Factors considered when prescribing.

- Information shared with patients.

- Physical health monitoring.

- Patient-led reduction/switching/withdrawal of APs.

This section was adapted for non-prescribing nurses to explore their role in supporting the prescribing of APs.

Experiences of and views on switching antipsychotics

- Current practice.

- Experiences of switching.

- Reasons for switching.

- When to consider switching.

- Views on and comfort when switching.

- Patient's responses.

- Barriers and facilitators to switching to improve physical health.

This section was adapted for non-prescribing nurses to explore their experience of support with switching APs.

Other interventions and support to promote physical health

- What other interventions are important.

- Involvement of patient's support networks.

Qualitative research' checklist was used to ensure rigour of analysis and credibility of findings. ${ }^{19}$

\section{Participants and recruitment}

Purposive sampling guided recruitment. Inclusion criteria were broad to include a diverse range of experiences from HCPs involved in prescribing APs to individuals with SMI. See online supplemental file 1 for an overview of HCP roles. GP practices across the West Midlands and North West of England were contacted. Psychiatrists and mental health nurses were identified through Midlands Partnership NHS Foundation Trust (MPFT). Study information was shared by email via existing contacts within the team, GP practice managers, and with staff in the Psychosis Pathways and Early Intervention Teams at MPFT. Sixteen GP practices, 21 psychiatrists and 53 nurses were contacted. As GPs are self-employed, they were offered reimbursement of $£ 80$ per hour (British Medical Association rates).

\section{Data collection}

A flexible topic guide (box 1) was developed using existing literature, the teams' clinical experience and with a patient and public involvement and engagement (PPIE) group.

All interviews were completed by AN (female research assistant, with qualitative research expertise) between January and August 2019. After two pilot interviews, the topic guide and interviews were discussed by AN and CAC-G. Following discussion in the team, the topic guide was modified iteratively as data were collected and analysed to facilitate further exploration of key themes. Prompts were added to explore: communication and responsibility between primary and secondary care, HCPs comfort with switching and patient's requests to switch. 
The majority of interviews were conducted face-to-face at the participant's place of work; one was completed by telephone. No repeat interviews were conducted. Informed consent was obtained prior to interviews, which were audio recorded and transcribed verbatim by an external transcription company or AN. Transcripts were anonymised and labelled with a unique participant identifier. The transcripts were not returned to participants for checking due to time constraints, however participants could withdraw data up to 2 weeks after the interview.

Data collection and analysis were conducted concurrently, informed by inductive thematic saturation and ended when no new codes or themes were identified. ${ }^{20}$ AN kept field notes that were discussed in research team meetings.

\section{Data analysis}

A thematic analysis was applied, led by the first author. ${ }^{21}$ AN read, re-read and coded all transcripts manually to identify key codes and preliminary themes. CAC-G and $\mathrm{SF}$ analysed a selection of the transcripts. The team drew upon clinical experiences to inform interpretations of the data. Preliminary themes were discussed during team meetings to explore similar and differing interpretations of the data before collaboratively agreeing the key themes. Constant comparison was used to analyse similarities and differences between participants' accounts to ensure themes captured different perspectives. ${ }^{22}$

\section{Patient and public involvement}

A McPin Foundation PPIE group contributed to topic guide development. Members of the Patient and Carer Involvement in Research group at MPFT reviewed preliminary analysis and contributed their interpretations of data presented.

\section{RESULTS}

Interviews were conducted with 9 GPs, 10 psychiatrists and 4 mental health nurses. Demographic characteristics of the participants are reported in table 1 .

Interviews ranged in duration from $44 \mathrm{~min}$ to $85 \mathrm{~min}$.

Five main themes were identified as follows: (1) priority given to mental health; (2) contrasting knowledge of antipsychotic medication (AP) risk profiles; (3) switching APs as a reactive intervention; (4) whose responsibility? and (5) the way forward: a collaborative approach. Each theme is described with illustrative data extracts, which include identifiers (GP, general practitioner; NU, mental health nurse; PS, psychiatrist).

\section{Priority given to mental health}

HCPs described the challenge of balancing the mental versus physical health needs of an individual. Indeed, some HCPs felt they were faced with an absolute choice:

I think it's which is the best of the two evils...you know is it, is it better that they've got some physical health problems but their mental health is so much better,

\begin{tabular}{|c|c|c|c|}
\hline Participant ID & Gender & Role & Time in role \\
\hline GP001 & Male & Salaried GP & 28 years \\
\hline GP002 & Male & Salaried GP & 3 years \\
\hline GP003 & Female & GP partner & 4 years \\
\hline GP004 & Female & Salaried GP & 29 years \\
\hline GP005 & Male & GP partner & 10 years \\
\hline GP006 & Male & GP partner & 8 years \\
\hline GP007 & Female & Salaried GP & 9 years \\
\hline GP008 & Female & Salaried GP & 8 years \\
\hline GP009 & Female & Salaried GP & 6 months \\
\hline NU001 & Male & $\begin{array}{l}\text { Community mental } \\
\text { health nurse }\end{array}$ & 2 years \\
\hline NU002 & Female & $\begin{array}{l}\text { Community mental } \\
\text { health nurse }\end{array}$ & 2 years \\
\hline NU003 & Female & $\begin{array}{l}\text { Community mental } \\
\text { health nurse }\end{array}$ & 40 years \\
\hline NU004 & Female & $\begin{array}{l}\text { Community mental } \\
\text { health nurse }\end{array}$ & 28 years \\
\hline PS001 & Male & $\begin{array}{l}\text { Consultant } \\
\text { psychiatrist }\end{array}$ & 3 years \\
\hline PS002 & Male & $\begin{array}{l}\text { Consultant } \\
\text { psychiatrist }\end{array}$ & 15 years \\
\hline PS003 & Male & $\begin{array}{l}\text { Specialty doctor in } \\
\text { psychiatry }\end{array}$ & 6 years \\
\hline PS004 & Male & $\begin{array}{l}\text { Consultant } \\
\text { psychiatrist }\end{array}$ & 22 years \\
\hline PS005 & Male & $\begin{array}{l}\text { Locum consultant } \\
\text { psychiatrist }\end{array}$ & 11 years \\
\hline PS006 & Female & Specialist doctor & 7 years \\
\hline PS007 & Female & Specialist doctor & 7 years \\
\hline PS008 & Female & $\begin{array}{l}\text { Specialty trainee } \\
\text { (ST5) }\end{array}$ & 1 year \\
\hline PS009 & Female & $\begin{array}{l}\text { Consultant } \\
\text { psychiatrist }\end{array}$ & 15 years \\
\hline PS010 & Male & $\begin{array}{l}\text { Consultant } \\
\text { psychiatrist }\end{array}$ & 15 years \\
\hline
\end{tabular}

GP, general practitioner; NU, mental health nurse; PS, psychiatrist.

and they can live their life and get a better quality of life, or is it better that they have no physical health problems and their mental health is out of control and they feel tortured. (NU004)

Mental health was prioritised by most HCPs due to the overt risk presented. Physical health risks were considered longer term, not requiring immediate attention:

We still tend to focus on the disease management first and then the illness management, the symptom management that's, that's our priority. I think the... sideeffects erm are still a second thought. (PS010)

Reflecting this priority, many HCPs described considering switching APs only to improve mental health not physical health: 
But that's the impression I get, is that the changing in antipsychotics is much more mental health driven than, than physical health driven. (GP006)

HCPs described improvements in individual's mental health as a long journey for themselves and patients. HCPs reported a reluctance to risk the stability achieved and associated improvements in patient's quality of life, relationships and well-being:

Once you've established somebody on medication that has treated their mental disorder, which has often taken a while to sort of be resolved and has caused lots of difficulties, I think there's still a tendency for us not to want to rock the boat...there's also a sense of relief that you know, we've dealt with their mental health, it's been a long journey but it's happened and that then trying to address the physical health can seem like an extra sort of challenge which we may feel that we don't want to look at. (PS004)

HCPs suggested patients also prioritise their mental health over physical health. HCPs faced a dilemma over whether to intervene when they observed deteriorations in patients' physical health, but perceived that patients were accepting of this deterioration due to not wanting to risk improvements in their mental health:

I think that's an area that's problematic is that someone who comes to see you that's putting on weight... you know maybe getting some mild diabetes as a result but their mental health is very well controlled... just because I'm putting on some weight, so what? And I've got some mild diabetes, so what? I feel great you know. This is the best drug that I've ever been on and I never want to go back to that two years of hell you know that I went through to get to this point so... it's a tricky area. (GP006)

\section{Knowledge about AP risk profiles}

All HCPs reported an awareness of some of the physical health side-effects of APs:

So we know from antipsychotic medication there's an increased risk of cardiovascular disease and diabetes, generally weight gain. (GP002)

But most GPs were unaware that certain APs carried higher metabolic risk than others:

I wasn't particularly aware of risk of one antipsychotic more than another in terms of metabolic risk. (GP007)

Specialist care HCPs were aware of levels of risk associated with different APs:

Olanzapine, clozapine, quetiapine, amisulpride they all cause weight gain. Risperidone maybe not as much as olanzapine. Aripiprazole shouldn't really cause weight gain but I've seen some people gain weight on aripiprazole. And lurasidone is marketed as one of those that shouldn't cause weight gain. (PS008)

Medication history, patient's preference, HCP's preference and views on efficacy and Trust formularies were the key factors considered during decision-making about prescribing lower risk APs:

Olanzapine has got a bit of a tendency of putting on weight, but it is still considered as a very effective medication, most, amongst all antipsychotic medication, my personal choice is that frankly that olanzapine is the most effective. (PS003)

Some psychiatrists reported a recent shift in their prescribing behaviours towards favouring lower risk APs to try and minimise longer-term impacts on physical health.

I think it's changing, my practice is changing so I'm much more mindful of the side-effects now than I was, not short term but longer term. And I think my prescribing is reflecting that. Even if perhaps it takes a bit longer for say aripiprazole to become effective I'm more patient now in continuing with a drug that may not be effective because of that lower risk of sideeffects. (PS010)

This change in prescribing was noted by mental health nurses who reported that more patients on their caseload were being prescribed lower risk APs, such as aripiprazole:

I've got quite a few people on aripiprazole, which has got a very low side-effect profile so that's quite positive. (NU004)

However, GPs reported the majority of people with psychosis were prescribed high-risk APs.

Quetiapine, but yeah to be fair the other one is olanzapine, they're the ones we see most but we do see some risperidone... and then the other one is aripiprazole, but very rarely do we see people on that. (GP003)

All secondary care HCPs were aware that switching from higher metabolic risk APs to lower risk APs could be used to address physical health problems; in contrast, the majority of GPs were unaware of this:

It's not something I was particularly aware of [laughs], but from this I'm now going to think more about whether there may be particular drugs that may be better in terms of their metabolic risk profile than others. (GP007)

GP prescribing and consideration of switching may be influenced by the idea that all APs pose a high metabolic risk and inevitably contribute to physical health conditions. 


\section{Switching as a reactive intervention}

Many HCPs were unsure at what point to consider switching APs to improve physical health. Where switching was considered, it was described as a reaction to an emerging physical health problem and/or following prompting by the patient but rarely as a proactive intervention. Ambiguity about how to best use routinely collected physical health data to inform and justify a switch, was reported:

At what point should switching be considered? You know, is it the point where HBA1C is at a certain level? Is it the point where they have put on so much weight? Is it at the point that some, you know, other physical parameters can be measured, resulting in yellow or red flags and that's when switching should occur so you don't wait until they need statins, you do it much sooner than that. (PS004)

The development of a new physical health condition acted as a key driver to prompt considerations about switching:

Once they develop diabetes, we need to look at an alternative. (PS006)

However, other HCPs felt that rather than consider switching an AP, they would increase or add further medication for the physical condition:

Is the answer switching? I've got to admit that... it's probably not the thing that's at the top of my list. You know if their diabetes has got worse I, as a doctor, would probably be more inclined to be going increase this dose of your anti-diabetic medication [laughs] or we need to add another anti-diabetic medication. (GP006)

Lifestyle advice was also considered by most HCPs as a first-line intervention for emerging cardiovascular and metabolic problems:

I'd probably look at lifestyle interventions because some of them have been really stable on their medication and if there are other things that we can do to address difficulties then I normally would say let's try that first and if it doesn't really work then we can look at the medication. (PS008)

Some HCPs described how patients had prompted consideration of a switch by raising their concerns about their physical health and/or AP side-effects:

I think it was the point that the service user said, actually 'I'm, I want to do something about this...' so if they hadn't said that, I suppose we would sort of notified it but maybe we wouldn't have been prompted to make a change without their erm, sort of prompt. (PS004)

Furthermore, some HCPs reported it was only when the patient linked physical health changes to APs that a medication review was considered:
Well if someone's coming in and saying - this patient comes in and says 'I've been on this and now look, I've put like a stone on, what's going on?', I'd be saying 'Yeah okay, I agree with you'. I'd write to the specialist to say 'You do realise what's going on?' (GP001)

\section{Whose responsibility?}

APs should be initiated in specialist care and then, once the patient's mental health is stable, primary care may take over prescribing. ${ }^{16}$ However, differing opinions were held about clinical roles and responsibilities around prescribing.

Secondary care HCPs observed an inconsistent approach in primary care, which often depended on locality. In some localities, decisions made at commissioning level prevented particular GP practices from prescribing APs:

Some of them [GPs] are very good to be honest, they do everything and they do the annual health check and give written information, they're excellent. Some are like 'we don't want to touch it, it's not our job...' There's a lot of reluctance from some of them. I think cost is also a factor for them. (PS009)

Some GPs described a diminished sense of responsibility over prescribing decisions for people with SMI, and the potential side-effects of APs, partly because they did not initiate the medication:

I'm not sitting down and thinking I might make this patient into a diabetic. That isn't really crossing my mind because that decision of prescribing has not been mine. (GP001)

Some GPs described being willing to continue to prescribe medication initiated by specialists, partly due to the perceived risk of making alterations:

Maybe we do sort of more easily just say 'yes, continue' to those sorts of medications that have been initiated by a specialist at some point than you would do about another medication that feels less risky to consider stopping potentially or changing. (GP007)

GPs reported that they would contact HCPs in secondary care if patients presented physical health problems linked to APs, rather than make changes in medication themselves:

I'd treat the underlying condition, you know, the diabetes, the cholesterol and address the weight. But if we thought it was due to the metabolic effects of the drugs, I'd involve the psychiatrist involved in their care. (GP004)

Another contested area of responsibility was identified around who should inform patients about side-effects of AP medications. Several GPs reported taking over prescribing responsibilities without meeting patients and 
discussing side-effects, based on the assumption patients had been informed in secondary care.

I rely on the psychiatrist to have already informed [the patient]. So I don't. You would expect them to have done that, he's taking responsibility for asking that drug to be provided so.... (GP001)

Many HCPs in primary and secondary care believed the responsibility for prescribing APs should be shared but that this was not currently the case:

Ideally it should be partnership working, collaborative working. I think primary care have an important role because...I think they need to be alert to understanding that if one of their patients is on antipsychotic medication, there are important physical parameters that they should be alert to...I think the responsibility very much falls to us as the prescribers of antipsychotic medication to at least cover those particular parameters and if then we detect abnormalities, whether it's in the ECG or cholesterol or diabetes, then involve the GP with the next step in terms of treatment or assessment...But it does cause tension sometimes. (PS004)

\section{The way forward: a collaborative approach}

Collaboration between clinicians, services and patients was thought to be key in supporting people with SMI. This would include discussion about: identifying the need for a switch, multidisciplinary discussion, supporting patients to make an informed decision about whether to switch, and creating a monitoring plan within teams:

I think with the help and support of our team that is the major thing that can help us for the switching, because we've got quite a few staff, you know, like we've got supportive, we've got CPN, support workers, they are monitoring them...community pharmacists, so they can help us to make a decision switching so it's like a team decision, it's not only one person's decision and obviously the patient's, the patient's decision at the end. (PS003)

GPs suggested that they couldn't offer this level of support to patient due to limited time and resources and this was a key reason for asking for support from secondary care:

If she's really deteriorating she needs a team around her anyway and so...it's not just about the drugs, it's about giving her wider support and getting the key worker involved so I suppose I'd get them... I'd just want more help in general rather than just with medication. (GP003)

Information sharing was felt to be crucial to supporting a switch of APs. HCPs in secondary care reported providing detailed information to GPs when handing over prescribing responsibility, including side-effects, risks and monitoring requirements:
And the GPs are prescribing we send them an ESCA with all the information about the side effects, the monitoring that's required, what dosage the patient is on and you know what were the last tests done so the GPs can carry through from that. (PS009)

However, some GPs reported receiving scant information when taking over prescribing; this left some GPs unsure about whose responsibility this was, what to monitor and how to use routinely collected data to inform clinical decisions.

They don't really give us any guidance on when do we worry, so we are measuring it but we don't... if we then identify it really high, we still don't know what to do and if you ask us specifically they just sort of go, 'yeah, whatever...' [laughs] so we've not had a huge amount of support I'd say in terms of managing the abnormalities. (GP003)

\section{DISCUSSION}

\section{Summary}

This qualitative study suggests that HCPs prioritised the mental health needs of patients with SMI over their physical health needs when making decisions about treatments, such as switching antipsychotics. Decisions that were felt to potentially jeopardise mental health stability and risk the onset or worsening of symptoms, were avoided, even at the detriment of increasing cardiometabolic risk. Levels of awareness about the risk profiles of APs differed; HCPs in secondary care had a greater understanding than GPs. Switching was rarely considered a proactive option; more often switching was reactive to the development of a new physical health condition. In some situations, it took patients raising concerns about their physical health and linking this to APs for HCPs to consider switching. Responsibility for decision-making around APs was commonly deferred to specialist care. The need for better collaboration was highlighted but the fragmentation of care, poor communication and inconsistencies in information-sharing prevented this, which may lead to duplication or gaps between primary and secondary care.

\section{Strengths and limitations}

The study provides an in-depth exploration of the barriers and facilitators to switching APs in primary and secondary care. Data analysis was rigorous and involved researchers from different clinical and academic backgrounds. The concept of inductive thematic saturation guided data collection and analysis. Saturation was reached with GP and psychiatrist data. Recruitment of mental health nurses was challenging, as many declined due to workload and/ or did not perceive switching as part of their clinical remit (which in itself is an important finding); saturation could not therefore be achieved in data arising from this group. The sample was self-selected, therefore participating 
clinicians may be more in favour of switching, although we were not able to assess this as treatment preferences were not explored. Psychiatrists and nurses were recruited from one mental health trust; further research is required to explore the themes reported in this paper in different geographic and service-level contexts. The research team attempted to recruit GPs working in localities where there was a blanket rule not to prescribe APs to explore barriers to collaborative working in the context of SMI, but no GPs agreed to be interviewed.

\section{Comparison with existing literature}

Almost all literature on switching APs focuses on strategies for switching and pharmacological interactions or pharmacokinetics. This is the first study to examine the views of HCPs in switching APs, hence the comparison with existing literature is difficult.

Levels of knowledge about the risk profiles of APs contrasted sharply between HCPs in primary and secondary care with the latter evidencing far greater awareness. However, people with SMI have previously described a lack of acknowledgement from psychiatrists about the negative impacts of medication on their quality of life and physical health concerns. ${ }^{23}$ Patients value the importance of good physical health, but often feel overwhelmed by comorbid health problems, ${ }^{24}$ and may feel powerless in decision-making about APs and consider APs to be the 'least worst option' despite problematic side-effects. ${ }^{23}$ HCPs in our study did not seem to engage patients in all aspects of AP decision-making, devaluing the insights that patients have. ${ }^{25}$ Exploration of patient perspectives on switching is needed to understand how shared decision-making can be achieved.

Switching presents one method to address physical health conditions in individuals with SMI. Other strategies include lifestyle interventions, choosing lower risk APs at treatment initiation and prescribing medications to treat physical health conditions. ${ }^{10}{ }^{16}$ Trial evidence of lifestyle interventions for people with SMI is poor for physical health outcomes and cost-effectiveness, ${ }^{8} 9$ although a recent trial showed that an 18-month intensive intervention could reduce overall cardiovascular risk. ${ }^{26}$ HCPs in our study reported continuing to promote lifestyle advice first-line. Concerns about jeopardising mental health presented a barrier to switching; evidence for the effectiveness of lower risk APs suggests these concerns may be exaggerated ${ }^{11-13}$ and should be reconsidered given the potential to achieve physical health improvements. ${ }^{15}$ The evidence on efficacy of $\mathrm{APs}^{27}$ and effects of APs on glucose levels ${ }^{28}$ clearly show that different APs have relatively small differences in efficacy for symptom management but significant differences for weight gain and metabolic side-effects.

A lack of clarity and consistency regarding where responsibility lies for identifying and managing physical problems in people with SMI remains a challenge. ${ }^{29}$ Previous research has highlighted challenges facing GPs prescribing APs for older people with dementia due to a lack of appropriate clinical guidance to inform decisions. ${ }^{30}$ A lack of collaboration between specialist mental health services and primary care have been reported elsewhere as a key barrier to the management of people with SMI. ${ }^{31}$ Pedley $e t a l^{25}$ call for mandated prescriber responsibilities to ensure that the input from all stakeholders is valued in decisions about AP medication. Our study further reiterated barriers of communication, information sharing and involvement of primary and secondary HCPs as part of multidisciplinary teams.

\section{Implications for practice and future research}

Given the limited evidence for brief lifestyle interventions to reduce cardiometabolic risk for people with SMI, HCPs should consider physical health at every contact, and switching APs should be on the agenda before cardiometabolic complications emerge. Guidance is needed to inform HCPs about how to use routinely collected physical health data to inform decision-making about interventions. Prompts in medical record systems could alert HCPs about key data and/or high metabolic risks related to APs. Identifying HCP training needs and providing further education is required to support switching. Existing tools that support the assessment of cardiometabolic health in people with SMI, such as the Lester tool, ${ }^{32}$ could be put to better use. A multidisciplinary team approach is needed that comprises clear lines of responsibility for HCPs that places patients at the centre of decision-making. Resources should be available to patients about AP risk profiles to support dialogue with HCPs; pharmacists could also play an important role here. Future research should explore patient views on switching.

These qualitative insights could inform the development of information and education resources for HCPs and patients and/or interventions (such as a switching protocol) for future evaluation and testing. Switching APs could offer a valuable means of responding to the widening mortality gap for people with SMI.

Acknowledgements We acknowledge the valuable input made by members of the McPin Foundation for contributing patient and public perspectives on the study design and advised on the conduct of this research. We also acknowledge and thank members of the Patient and Carer Involvement in Research group at MPFT for contributing to the analysis during the study. We also acknowledge and thank the HCPs who kindly agreed to be interviewed.

Contributors The study was conceived by SF and CAC-G who wrote the application for funding and the protocol. AN collected data. All authors (AN, TK, SF, JT and CAC-G) contributed to data analysis. TK and AN drafted the manuscript and all authors commented on the manuscript.

Funding This work was supported by a research grant from Sunovion Pharmaceuticals. CAC-G is also part-funded by West Midlands Applied Research Collaboration.

Competing interests Sunovian Pharmaceuticals funded this work; however, the conduct of the research, data collection, analysis and drafting of this manuscript were completed independently by the research team. No members of the research team have a financial relationship with Sunovian Pharmaceuticals.

Patient consent for publication Not required.

Ethics approval The study received ethical approval from Keele University Research Ethics Committee (ref: RG-0177-17) and the Health Research Authority (IRAS ID: 231154). 
Provenance and peer review Not commissioned; externally peer reviewed.

Data availability statement Data are available from the corresponding author on reasonable request.

Supplemental material This content has been supplied by the author(s). It has not been vetted by BMJ Publishing Group Limited (BMJ) and may not have been peer-reviewed. Any opinions or recommendations discussed are solely those of the author(s) and are not endorsed by BMJ. BMJ disclaims all liability and responsibility arising from any reliance placed on the content. Where the content includes any translated material, BMJ does not warrant the accuracy and reliability of the translations (including but not limited to local regulations, clinical guidelines, terminology, drug names and drug dosages), and is not responsible for any error and/or omissions arising from translation and adaptation or otherwise.

Open access This is an open access article distributed in accordance with the Creative Commons Attribution Non Commercial (CC BY-NC 4.0) license, which permits others to distribute, remix, adapt, build upon this work non-commercially, and license their derivative works on different terms, provided the original work is properly cited, appropriate credit is given, any changes made indicated, and the use is non-commercial. See: http://creativecommons.org/licenses/by-nc/4.0/.

\section{ORCID iD}

Tom Kingstone http://orcid.org/0000-0001-9179-2303

\section{REFERENCES}

1 Chesney E, Goodwin GM, Fazel S. Risks of all-cause and suicide mortality in mental disorders: a meta-review. World Psychiatry 2014;13:153-60.

2 Hayes JF, Marston L, Walters K, et al. Mortality gap for people with bipolar disorder and schizophrenia: UK-based cohort study 2000-2014. Br J Psychiatry 2017;211:175-81.

3 Stubbs B, Vancampfort D, De Hert M. The prevalence and predictors of type two diabetes mellitus in people with schizophrenia: a systematic review and comparative meta-analysis. Acta Psychiatr Scand 2015;132:144-57.

4 Correll CU, Solmi M, Veronese N, et al. Prevalence, incidence and mortality from cardiovascular disease in patients with pooled and specific severe mental illness: a large-scale meta-analysis of $3,211,768$ patients and $113,383,368$ controls. World Psychiatry 2017;16:163-80.

5 Vancampfort D, Correll CU, Galling B, et al. Diabetes mellitus in people with schizophrenia, bipolar disorder and major depressive disorder: a systematic review and large scale meta-analysis. World Psychiatry 2016;15:166-74.

6 Reilly S, Olier I, Planner C, et al. Inequalities in physical comorbidity: a longitudinal comparative cohort study of people with severe mental illness in the UK. BMJ Open 2015;5:e009010.

7 NHS England. Implementing the five year forward view for mental health. London: NHS England, 2016. https://www.england.nhs.uk/ wp-content/uploads/2016/02/Mental-Health-Taskforce-FYFV-final. pdf

8 Osborn D, Burton A, Hunter R, et al. Clinical and cost-effectiveness of an intervention for reducing cholesterol and cardiovascular risk for people with severe mental illness in English primary care: a cluster randomised controlled trial. Lancet Psychiat 2018;5:145-54.

9 Holt RIG, Gossage-Worrall R, Hind D, et al. Structured lifestyle education for people with schizophrenia, schizoaffective disorder and first-episode psychosis (STEPWISE): randomised controlled trial. $\mathrm{Br}$ $J$ Psychiatry 2019;214:63-73.

10 Cooper SJ, Reynolds GP, Barnes TRE, et al. BAP guidelines on the management of weight gain, metabolic disturbances and cardiovascular risk associated with psychosis and antipsychotic drug treatment. J Psychopharmacol 2016;30:717-48.

11 Leucht S, Cipriani A, Spineli L, et al. Comparative efficacy and tolerability of 15 antipsychotic drugs in schizophrenia: a multipletreatments meta-analysis. Lancet 2013;382:951-62.
12 Bai Z, Wang G, Cai S, et al. Efficacy, acceptability and tolerability of 8 atypical antipsychotics in Chinese patients with acute schizophrenia: a network meta-analysis. Schizophr Res 2017;185:73-9.

13 Pillinger T, McCutcheon RA, Vano L, et al. Comparative effects of 18 antipsychotics on metabolic function in patients with schizophrenia, predictors of metabolic dysregulation, and association with psychopathology: a systematic review and network meta-analysis. Lancet Psychiat 2020;7:64-77.

14 Marston L, Nazareth I, Petersen I, et al. Prescribing of antipsychotics in UK primary care: a cohort study. BMJ Open 2014;4:e006135.

15 Mukundan A, Faulkner G, Cohn T, et al. Antipsychotic switching for people with schizophrenia who have neuroleptic-induced weight or metabolic problems. Cochrane Database Syst Rev 2010;12:CD006629.

16 NICE. Psychosis and schizophrenia in adults: prevention and management. Clinical guideline CG178, 2014. Available: https:// www.nice.org.uk/guidance/cg178/ifp/chapter/Antipsychoticmedication

17 NHS England. Improving physical healthcare for people with living with severe mental illness in primary care, 2018. Available: https:// www.england.nhs.uk/wp-content/uploads/2018/02/improvingphysical-health-care-for-smi-in-primary-care.pdf

18 Sim J, Wright C. Research in health care: concepts, designs and methods. Nelson Thornes, 2000.

19 Tong A, Sainsbury P, Craig J. Consolidated criteria for reporting qualitative research (COREQ): a 32-item checklist for interviews and focus groups. Int J Qual Health Care 2007;19:349-57.

20 Saunders B, Sim J, Kingstone T, et al. Saturation in qualitative research: exploring its conceptualization and operationalization. Qual Quant 2018;52:1893-907.

21 Braun V, Clarke V. Using thematic analysis in psychology. Qual Res Psychol 2006;3:77-101.

22 Charmaz K. Constructing grounded theory. London: Sage, 2014.

23 Morant N, Azam K, Johnson S, et al. The least worst option: user experiences of antipsychotic medication and lack of involvement in medication decisions in a UK community sample. J Ment Health 2018;27:322-8.

24 Cocoman AM, Casey M. The physical health of individuals receiving antipsychotic medication: a qualitative inquiry on experiences and needs. Issues Ment Health Nurs 2018;39:282-9.

25 Pedley R, McWilliams C, Lovell K, et al. Qualitative systematic review of barriers and facilitators to patient-involved antipsychotic prescribing. BJPsych Open 2018;4:5-14.

26 Daumit GL, Dalcin AT, Dickerson FB, et al. Effect of a comprehensive cardiovascular risk reduction intervention in persons with serious mental illness. JAMA Network Open 2020;3:e207247.

27 Huhn M, Nikolakopoulou A, Schneider-Thoma J, et al. Comparative efficacy and tolerability of 32 oral antipsychotics for the acute treatment of adults with multi-episode schizophrenia: a systematic review and network meta-analysis. Lancet 2019;394:939-51.

28 Zhang Y, Liu Y, Su Y, et al. The metabolic side effects of 12 antipsychotic drugs used for the treatment of schizophrenia on glucose: a network meta-analysis. BMC Psychiatry 2017;17:373.

29 De Hert M, Cohen DAN, Bobes J, et al. Physical illness in patients with severe mental disorders. II. Barriers to care, monitoring and treatment guidelines, plus recommendations at the system and individual level. World Psychiatry 2011;10:138-51.

30 Jennings AA, Foley T, McHugh S, et al. "Working away in that Grey Area...' A qualitative exploration of the challenges general practitioners experience when managing behavioural and psychological symptoms of dementia. Age Ageing 2018;47:295-303.

31 van Hasselt FM, Oud MJT, Loonen AJM. Practical recommendations for improvement of the physical health care of patients with severe mental illness. Acta Psychiatr Scand 2015;131:387-96.

32 Shiers DE, Rafi I, Cooper SJ. 2014 update (with acknowledgement to the late Helen Lester for her contribution to the original 2012 version) positive cardiometabolic health resource: an intervention framework for patients with psychosis and schizophrenia. London: Royal College of Psychiatrists, 2014. 